\title{
Red Queen strange attractors in host-parasite replicator gene-for-gene coevolution
}

\author{
Josep Sardanyés ${ }^{\mathrm{a}, *}$, Ricard V. Solé ${ }^{\mathrm{a}, \mathrm{b}}$ \\ a Complex Systems Lab (ICREA-UPF), Barcelona Biomedical Research Park (PRBB-GRIB), Dr. Aiguader 88, 08003 Barcelona, Spain \\ b Santa Fe Institute, 1399 Hyde Park Road, Santa Fe, NM 87501, USA
}

Accepted 16 August 2006

Communicated by Professor M.S. El Naschie

\begin{abstract}
We study a continuous time model describing gene-for-gene, host-parasite interactions among self-replicating macromolecules evolving in both neutral and rugged fitness landscapes. Our model considers polymorphic genotypic populations of sequences with 3 bits undergoing mutation and incorporating a "type II" non-linear functional response in the host-parasite interaction. We show, for both fitness landscapes, a wide range of chaotic coevolutionary dynamics governed by Red Queen strange attractors. The analysis of a rugged fitness landscape for parasite sequences reveals that fittest genotypes achieve lower stationary concentration values, as opposed to the flattest ones, which undergo a higher stationary concentration. Our model also shows that the increase of parasites pressure (higher self-replication and mutation rates) generically involves a simplification of the host-parasite dynamical behavior, involving the transition from a chaotic to an ordered coevolutionary phase. Moreover, the same transition can also be found when hosts "run" faster through the hypercube. Our results, in agreement with previous studies in host-parasite coevolution, suggest that chaos might be common in coevolutionary dynamics of changing self-replicating entities undergoing a host-parasite ecology.
\end{abstract}

(c) 2006 Elsevier Ltd. All rights reserved.

\section{Introduction}

The presence of chaos and other non-linear phenomena in continuous as well as in discrete ecological models has been shown up to now in a wide variety of systems [1-20]. Ecological systems seem to have all necessary ingredients for the appearance of chaotic dynamics $[1,2,10,19,21]$ as palpable non-linear couplings or multiple state variable. The presence of chaos in physical systems is nowadays practically indisputable. From the more abstract models $[22,23]$ to experimental confirmations [24-29], chaos seems to be the rule more than the exception in non-linear physics [30]. Other classic examples of chaotic dynamics are found in non-equilibrium chemical kinetics. For instance, chaos has been described in several theoretical models for chemical systems [31,32] as well as in experimental data for

\footnotetext{
${ }^{*}$ Corresponding author. Tel.: +34 933160532; fax: +34 932213237.

E-mail address: josep.sardanes@upf.edu (J. Sardanyés).
} 
the well known Belousov-Zhabotinskii reaction [33-35]. However, the plausible presence of chaos as well as its role in biological processes as ecological dynamics has generated and intense debate during the last decades $[1,2,6,12,18,20,36-38]$ (see also $[19,30,39]$ for reviews). For some authors chaos in single population dynamics might be synonymous of higher extinction probabilities [37]. Nevertheless many theoretical approaches give support to the opposite idea, where chaos might not involve population instabilities and extinction $[7,11,12,16]$. In this sense, it has been standed out that the presence of an attractor might involve the confinement of solutions (i.e. population sizes) in phase space thus including the possibility to have oscillatory dynamics far away from extinction [30]. Moreover strange attractors can be stable although (because of its dynamical properties) no equilibrium is approached [30]. Although it has been suggested for some authors that the clear detection of chaos in natural populations might be a difficult $[18-20,30,40]$ or even an impossible [41] task, several studies give support to the presence of chaos in natural ecosystems $[12,15,30,38,42,43]$.

Coevolutionary dynamics among interacting species has also been an important subject of research in many fields of biology as ecology or immunology $[4,5,7,11,15,16,44-47]$. It is well known that host-parasite, predator-prey or hostparasitoid interactions are ubiquitous in nature, playing an important role in ecosystems constituting the so-called ecological networks, and a large amount of theoretical approaches to give away the asymptotic dynamics of such systems have been developed $[3,5,7,10,11,17,48,49]$. The concept of gene-for-gene (GFG) coevolution has been applied to hostpathogen interactions in agriculture, natural plant populations and in mathematical models for coevolution [47]. The GFG hypothesis, originally formulated by Flor, states that "for each gene determining resistance in the host there is a corresponding gene for avirulence in the parasite with which it specifically interacts" [47]. This kind of coevolution has been described in several agricultural plant-pathogen associations, and it is thought that GFG interactions among plants and pathogens as viruses, bacteria and fungi are likely to be found in nature (see [47]).

The presence of oscillations governed by limit cycles $[18,44,50]$, as well as the presence of strange attractors $[4,7,10,11,16-18]$ have been described in theoretical models of interacting species. Such oscillatory behaviors in interacting population dynamics actually correspond to the so-called Red Queen dynamics. The elegant Red Queen hypothesis of dynamical evolution was initially introduced by Leigh Van Valen, who actually stated that such a law could be thought in terms of an unorthodox game theory [51]. This theory, which corresponds to a zero-sum game (where each species competes, directly or indirectly, with all others, and no species ever wins because new adversaries might replace the losers) [52], emphasizes the relevant role of biotic interactions in the coevolution of species above the abiotic factors [53], in an ecologically "driven" evolution.

Actually, as stated by Van Valen [51]: “. . . The Red Queen does not need changes in the physical environment, although she can accommodate them...". As previously mentioned, an important proposition of this theory is the assertion that the effective environment of each species consists predominantly of the other species in the ecosystem. Hence, evolutionary changes in one species involves a change in the effective environment of all the others. In this sense, by moving across a fitness landscape, populations changes that landscape, and each population can be part of the fitness landscape of the other [54]. Thus evolutionary responses as continuous evolution are required of all the affected species to maintain ("to keep in the same place") [53]. This is the idea behind what the Red Queen explained to Alice: "... Now here, you see, it takes all the running you can do, to keep in the same place...", as Carroll wrote [55]. Like Alice, each taxon must continuously evolve as rapidly as possible in order to survive [56]. The Red Queen hypothesis is also thought to play a relevant role in the appearance and persistence of sexual reproduction [57-60]. In this sense antagonistic interactions with parasites may select sexual reproduction as a way to increase genetic variability in order to reduce the risk of infection in offspring $[58,60]$.

In this work we numerically explore the dynamics of GFG coevolution among host-parasite self-replicating bit sequences by considering panmictic, polymorphic genotypic populations with ongoing mutation. Darwinian dynamics based on mutation and selection are the basis of mathematical models for adaptation and coevolution of biological populations [54]. Furthermore, as Stadler and Stadler [61] eloquently stated : “... The relevant interest in chemical reaction kinetics associated with replication processes is an indispensable prerequisite for any understanding of evolution at the molecular level...". Hence the analysis of the dynamical behavior of systems with self-replicating macromolecules undergoing host-parasite interactions and mutation might serve as a basis for understanding molecular evolutionary processes as well as to deep on fundamental processes of prebiotic evolution. Moreover, this models can also be generically applied to characterize ecological deterministic dynamics, in which mutation rates might represent the rates of evolution of reproductive (i.e. self-replicating) host-pathogen entities.

We here specifically use a continuous time model which includes a non-linear functional response in the host-parasite interaction and allows us to explore coevolutionary traits in both neutral and rugged fitness landscapes. The model considers two interacting species (one host and its parasite) where each species has eight possible gene states (alleles) defined as 3-bit sequences, which define two hypercubes that are actually coupled by means of the host-parasite ecology (see Fig. 1). Our aim is to deep in the underlying coevolutionary dynamics as well as in the role of both 


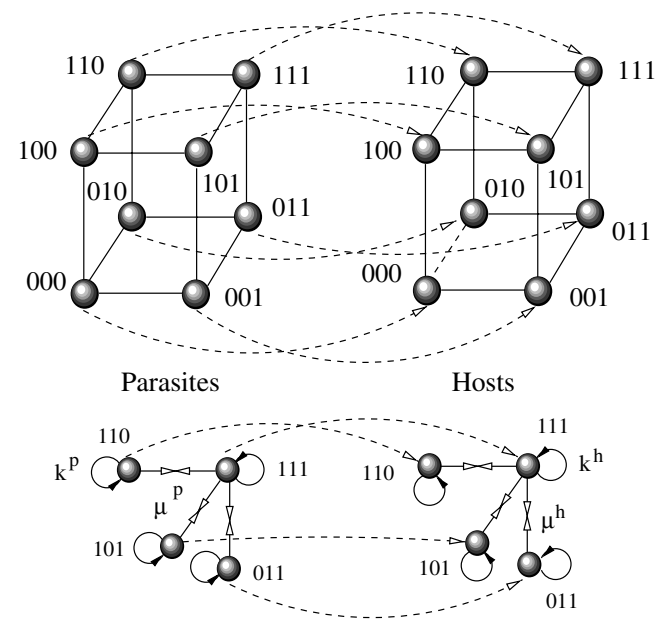

Fig. 1. Host-parasite interactions among self-replicating genotypes with mutation defining a three-dimensional sequence space $\left(\mathscr{H}^{v}\right)$ which represents a fitness landscape (here for the neutral case where the fitness is the same for all the genotypes). Below we show the dynamical processes happening in both hypercubes as mutation, proportional to mutation parameter, $\mu^{(\mathrm{h}, \mathrm{p})}$; and self-replication proportional to replication parameter, $k^{(\mathrm{h}, \mathrm{p})}$. Dashed lines indicate the host-parasite interaction assuming a perfect matching.

self-replication and mutation processes in the asymptotic dynamics of both populations under two different fitness landscapes.

\section{Host-parasite continuous model}

The mathematical model used to analyze the mass interaction kinetics of host-parasite replicators is given, assuming a perfect matching interaction i.e. $i \rightarrow i$ predation, by the next 16 th dimensional dynamical system according to

$$
\begin{aligned}
& \dot{x}_{i}=k_{i}^{\mathrm{h}} x_{i}\left(1-\sum_{j \in \mathscr{H}^{v}} x_{j}\right)-\Psi\left(x_{i}, y_{i}\right)+\frac{\mu_{i}^{\mathrm{h}}}{v}\left(\sum_{<j>_{i}} x_{j}-x_{i}\right)-\epsilon_{i}^{\mathrm{h}} x_{i}, \\
& \dot{y}_{i}=\Psi\left(x_{i}, y_{i}\right)+\frac{\mu_{i}^{\mathrm{p}}}{v}\left(\sum_{<j>i} y_{j}-y_{i}\right)-\epsilon_{i}^{\mathrm{p}} y_{i} .
\end{aligned}
$$

Here $x_{i}$ and $y_{i}$ denote, respectively, the concentration of the $i$ th host and of the $i$ th parasite genotype (with $\left\{x_{i}, y_{i}\right\} \in \mathscr{H}^{v}$ and $i=1 \ldots 2^{v=3}$ ) defining a $v$-dimensional sequence space, $\mathscr{H}^{v}$ (see Fig. 1). Here host genotypes have a logistic-like growth constraint in self-replication indicated in the first parenthesis of Eq. (1), being $\sum_{j \in \mathscr{H}^{v}} x_{j}$, the total host population. The term $\sum_{<j>i} x_{j}-x_{i}$, denotes the diffusion processes among hypercubic orthant neighbors. Moreover, $k_{i}^{\mathrm{h}}$ and $k_{i}^{\mathrm{p}}$ are self-replication parameters i.e. per capita growth rate, for host (h) and parasite (p) sequences; $\epsilon_{i}^{\mathrm{h}}$ and $\epsilon_{i}^{\mathrm{p}}$ are decay rates representing spontaneous hydrolysis processes. Finally, $\mu_{i}^{\mathrm{h}}$ and $\mu_{i}^{\mathrm{p}}$ denote, respectively, the mutation rates for both host and parasite genotypes. The term

$$
\Psi\left(x_{i}, y_{i}\right)=\frac{k_{i}^{\mathrm{p}} y_{i} \alpha x_{i}}{k_{i}^{\mathrm{h}}+\alpha x_{i}},
$$

denotes a Holling "type II" functional response in the host-parasite interaction where $\alpha$ is the encounter rate (hereafter $\alpha=1$ ). This expression is actually a saturating function for the consumption of prey (host) by predators (parasite), and has been used in ecological models $[10,49]$. Since the parameter space is extremely large we will study, as a first approach, the neutral fitness landscape with $k_{1}^{\mathrm{h}}=\cdots=k_{i}^{\mathrm{h}} \equiv k^{\mathrm{h}}$ and $k_{1}^{\mathrm{p}}=\cdots=k_{i}^{\mathrm{p}} \equiv k^{\mathrm{p}}, \forall i$.

The same analyses will be considered in a more real situation where parasite genotypes present different fitness properties and thus evolve in a rugged fitness landscape. We will specifically assume that parasite genotypes 000 and 111 have a different fitness value, $K^{\mathrm{p}}$, with $K^{\mathrm{p}}>k^{\mathrm{p}}$ or $K^{\mathrm{p}}<k^{\mathrm{p}}$, being $k^{\mathrm{p}}$ the self-replication rate of the other six genotypes of the sequence space. For both approaches we will also assume: (a) mutation rates are the same for all host genotypes as well as for parasite genotypes i.e. $\mu_{1}^{\mathrm{h}}=\cdots=\mu_{i}^{\mathrm{h}} \equiv \mu^{\mathrm{h}}$ and $\mu_{1}^{\mathrm{p}}=\cdots=\mu_{i}^{\mathrm{p}} \equiv \mu^{\mathrm{p}}$; (b) hosts evolve in a neutral fitness landscape i.e. $k^{\mathrm{h}}=1$; (c) and $\epsilon_{i}^{\mathrm{h}}=\epsilon_{i}^{\mathrm{p}}=10^{-2}$. 


\section{Analyses and results}

The dynamics of Eqs. (1) and (2) is investigated by means of numerical integration using the standard fourth-order Runge-Kutta method [21] with a constant time stepsize $\delta t=0.1$. We will assume genotypes extinction with concentrations lower than $10^{-8}$. As initial conditions we hereafter use higher concentration values for host genotypes, thus with low parasite concentrations.

\subsection{Neutral fitness landscape}

As above-mentioned, we firstly study the simplest i.e. neutral, fitness landscape where both species evolve. An example of chaotic coevolutionary dynamics is shown in Fig. 2. We specifically show the whole host-parasite dynamics (a), where the concentration for the parasite population is shown to achieve higher values. On the contrary, host concentration is lower but not near the extinction threshold. An overview to the local host dynamics (see Fig. 2(b)) shows that time trajectories achieve near extinction values, however their asymptotic dynamics is the confinement into the strange attractors also shown in Fig. 2. The inset in Fig. 2(b) shows the fingerprint of chaotic dynamics, that is, sensitive dependence on initial conditions. In the same figure we show the global attractor governing host-parasite coevolution. Note that the flow remains confined far away from the extinction point.

In order to display the array of dynamical behaviors for the most relevant parameters of the model as parasites selfreplication, $k^{\mathrm{p}}$, and host-parasite mutation rates, $\mu^{(\mathrm{h}, \mathrm{p})}$, let us now analyze the bifurcation diagrams by using such rates as control parameters. Fig. 3 shows a bifurcation diagram by using $k^{\mathrm{p}}$ as control parameter, in which a wide chaotic domain in the dynamics of a single genotype is found, the same dynamical pattern is followed by the other genotypes (results not shown). Here the increase of parasites pressure initially involves an increase of the host-parasite fluctuations. However from $k^{\mathrm{p}} \approx 0.2$ such a fluctuations starts decreasing in an exponential fashion. The role of mutation rates in the asymptotic dynamics is displayed in Fig. 4. We here specifically show three different cases, although in all of them chaos is found with low values of mutation rates, and the dynamics is generically simplified at increasing mutation rates. In Fig. 4(a) and (b) we use, respectively, a low and a high $k^{\mathrm{p}}$ value. In both cases the range of fluctuations is shown to decrease at increasing parasite mutation rates. Note that in Fig. 4(a), although $k^{\mathrm{p}}$ is low, hosts and parasites achieve higher population values as opposed to Fig. 4(b), where parasites pressure is higher and involves lower concentrations for host-parasite genotypes. In Fig. 4(c) we use hosts mutation rates as control parameter. Here, as previously men-

a

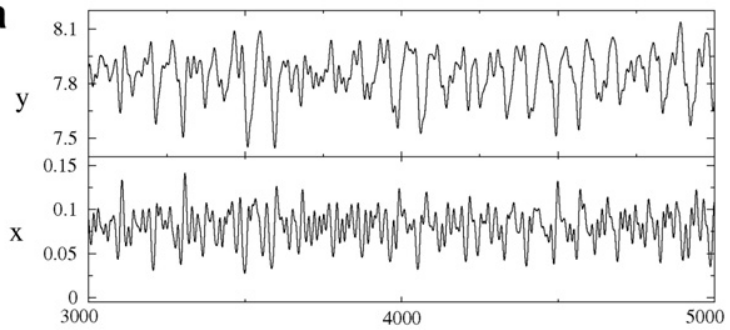

b

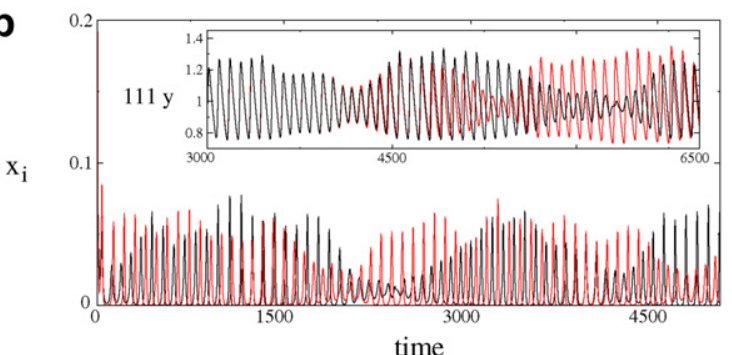

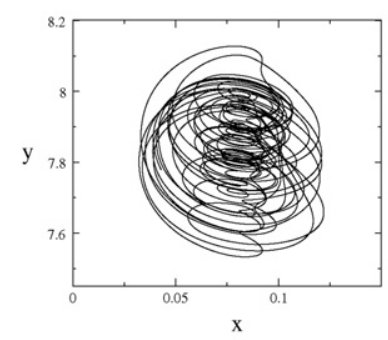
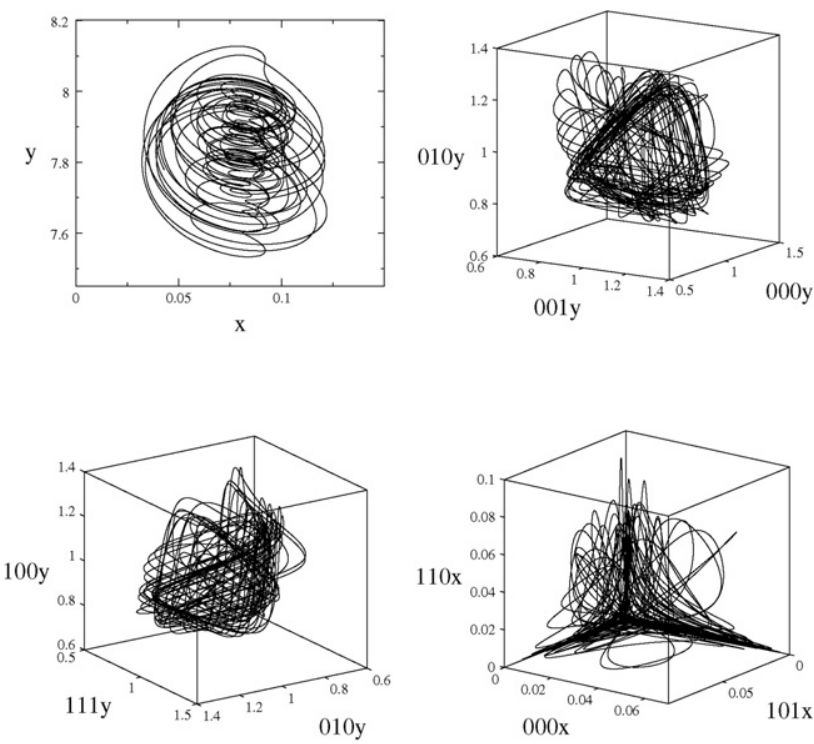

Fig. 2. Coevolutionary dynamics in the chaotic domain with $k^{\mathrm{p}}=0.95$ and $\mu^{\mathrm{h}}=\mu^{\mathrm{p}}=10^{-3}$ for the neutral fitness landscape. In (a) we show the global host-parasite time series and in (b) the dynamics for host genotypes 000 (black) and 011 (red). The inset in (b) displays the divergence of nearby trajectories in the chaotic domain for our model: in black we show a time trajectory for the parasite genotype 111 and initial condition $y_{111}(0)=\eta$, and in red we represent the same trajectory now with $y_{111}(0)=\eta+10^{-8}$ as initial condition. The phase portraits at the right show the Red Queen strange attractors for both global and local dynamics. (For interpretation of the references in colour in this figure legend, the reader is referred to the web version of this article.) 

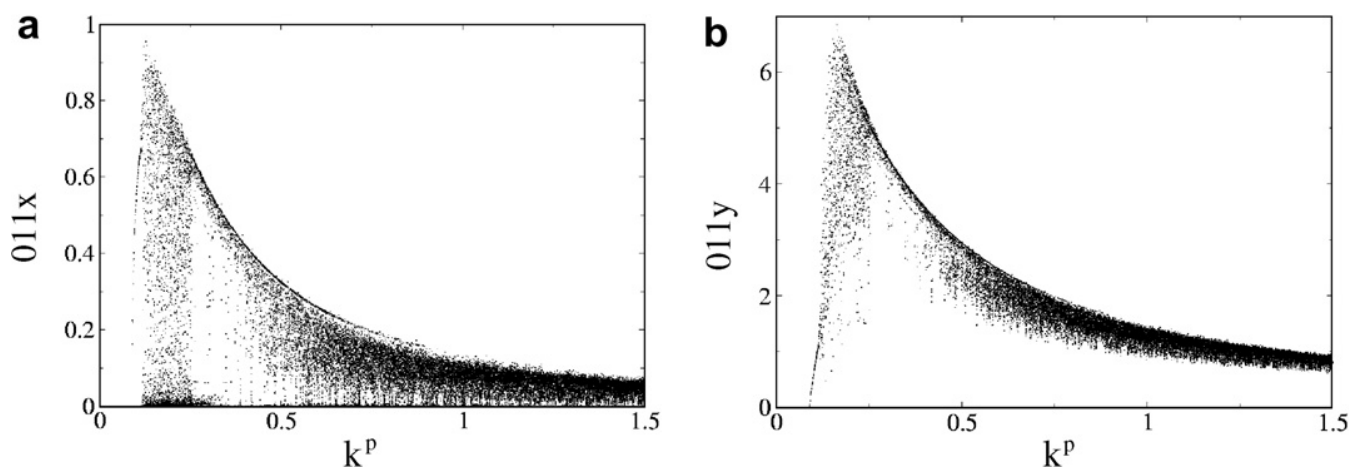

Fig. 3. Bifurcation diagrams with $k^{\mathrm{p}}$ as control parameter and $\mu^{\mathrm{h}}=\mu^{\mathrm{p}}=10^{-4}$ for the neutral fitness landscape. The graphs show the successive local maxima of a time series on the attractor $\left(15 \times 10^{3}\right.$ last time steps of a time series with $3 \times 10^{5}$ time steps) for the host (a) and parasite (b) genotypes 011 .

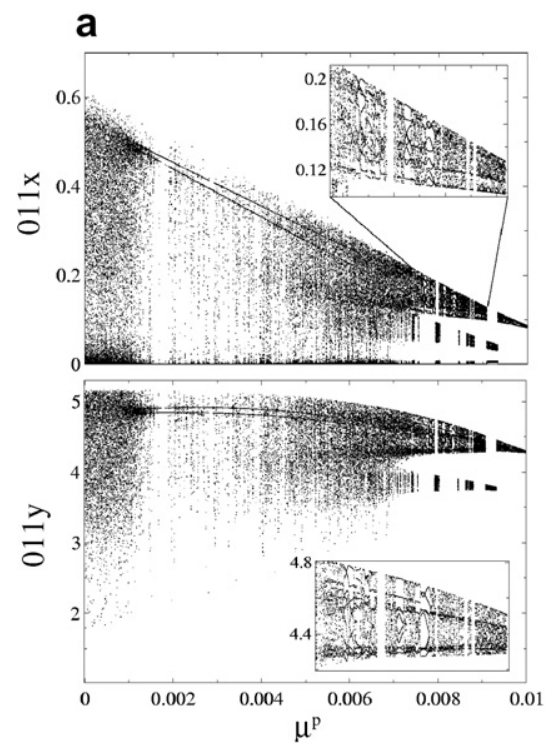

b

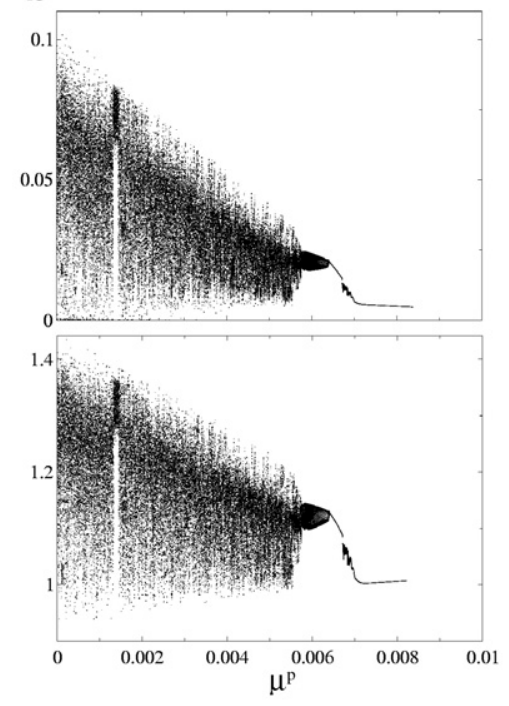

c

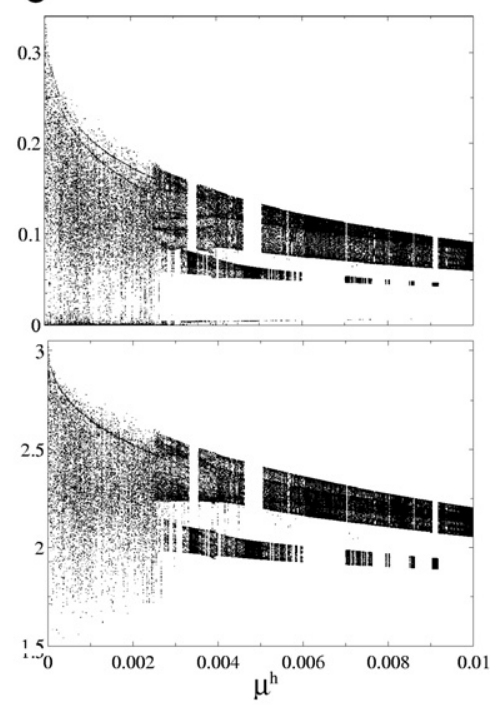

Fig. 4. As Fig. 3 now using mutation rates as control parameter. In (a) $k^{\mathrm{p}}=0.25$ and $\mu^{\mathrm{h}}=10^{-3}$, where both insets show the same enlarged view of a small part of the diagram. In (b) $k^{\mathrm{p}}=0.95$ and $\mu^{\mathrm{h}}=10^{-3}$; and in (c) $k^{\mathrm{p}}=0.5$ and $\mu^{\mathrm{p}}=10^{-3}$. Note that when both host and parasite mutation rates are high enough, the dynamics undergoes a transition from chaotic to quasiperiodic trajectories.

tioned, the chaotic domain is reduced to low mutation rates. Moreover in the previous two examples i.e. Fig. 4(a) and (b), chaos is also found with low $\mu^{\mathrm{p}}$ values, although the chaotic domain is larger as opposed to Fig. 4(c) (see Fig. 4 for details).

The global host-parasite dynamics with same rates of change for both host and parasite genotypes is summarized in Fig. 5(A). It is clearly shown that the increase in parasites self-replication involves, as seen in Fig. 3, an initial increase in parasite concentration and a decrease in host concentration. Note that with $k^{\mathrm{p}} \gtrsim 0.25$ both host-parasite concentrations decrease. The role of parasites mutation rate on the dynamics is shown in Fig. 5(B), where the concentration for both host-parasite populations is shown to decrease and increase, respectively. Here the decrease of $\mu^{\mathrm{p}}$ makes the flow in phase space to become turbulent. Note that the attractors become quasi-periodic when $\mu^{\mathrm{p}} \gtrsim 0.007$ (see Fig. 5 for details).

\subsection{Rugged fitness landscape}

The dynamics of coevolution in the rugged fitness landscape also displays a wide coevolutionary scenario which can be governed by strange attractors. In Fig. 6 we show the chaotic coevolution for this fitness landscape. Note that the 

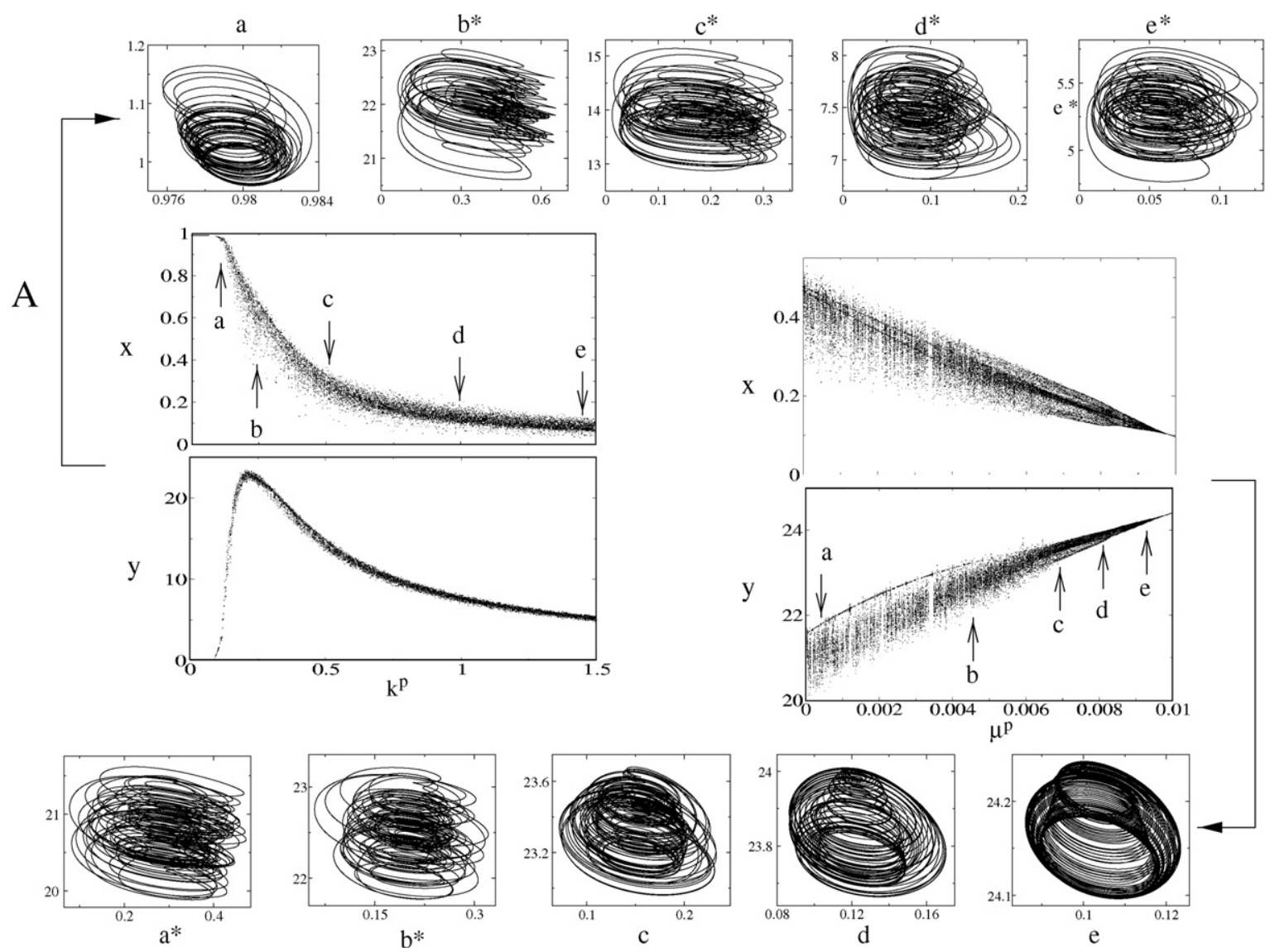

Fig. 5. Bifurcation diagrams for the global host-parasite dynamics using $k^{\mathrm{p}}$ and $\mu^{\mathrm{p}}$ as control parameters for the neutral fitness landscape. In (A) $\mu^{\mathrm{h}}=\mu^{\mathrm{p}}=10^{-4}$. In (B) $k^{\mathrm{p}}=0.3$ and $\mu^{\mathrm{h}}=10^{-3}$. In the upper part we show the topological evolution of the global host-parasite attractor for different values of $k^{\mathrm{p}}$ corresponding to the arrows in the bifurcation diagram: (a) $k^{\mathrm{p}}=0.1$, (b) $k^{\mathrm{p}}=0.25$, (c) $k^{\mathrm{p}}=0.5$, (d) $k^{\mathrm{p}}=1$ and (e) $k^{\mathrm{p}}=1.43$. Below we show the same now for parasites mutation rate with (a) $\mu^{\mathrm{p}}=4 \times 10^{-4},(\mathrm{~b})$ $\mu^{\mathrm{p}}=0.00448$, (c) $\mu^{\mathrm{p}}=0.0068$, (d) $\mu^{\mathrm{p}}=0.0083$ and (e) $\mu^{\mathrm{p}}=0.0093$. Note that the decrease in parasite mutation rate involves the transition from an ordered to a turbulent coevolutionary phase. Hereafter the asterisks will indicate the attractors showing sensitive dependence on initial conditions.

fittest parasite genotype i.e. 111 (in the inset) achieves a lower stationary concentration while two flattest parasite genotypes i.e. 100 and 010 , are shown to achieve a higher stationary concentration. This result is also visible in the dynamics of host genotypes. As an example we also show in Fig. 6, the time evolution of both host genotypes 000 (red) and 011 (black). Note that on average the sequence 011 undergoes larger fluctuations. In this figure we also show some projections in two-dimensional phase space, where such a phenomenon can also be visualized (see Fig. 6 for details).

The bifurcation diagrams of Fig. 7 display the array of dynamical behaviors at changing the ruggedness of the fitness landscape for parasite genotypes. Here we fix $k^{\mathrm{p}}=0.3$ and $\mu^{\mathrm{h}}=\mu^{\mathrm{p}}=10^{-4}$, and by tuning $K^{\mathrm{p}}$ we can follow the dynamics in two different scenarios: (i) the self-replication rate for both parasite sequences 111 and 000 is lower than the other six genotypes, thus these two sequences are flattest; (ii) parasite genotypes 111 and 000 self-replicate at a higher rates than the other six parasite genotypes, now becoming fittest. Scenarios (i) and (ii) are separated with the arrow (see Fig. 7). It is shown that fittest parasite genotypes fluctuate in a lower concentration range, and flattest genotypes fluctuate in a larger range of concentration values. The same phenomenon is also shown to happen for the homologous host sequences. In Fig. 7(b) we also show the bifurcation diagrams for the global host-parasite dynamics.

The role of parasite mutation rate on the dynamics is shown in Fig. 8. Here we focus in scenario (ii), with $K^{\mathrm{p}}>k^{\mathrm{p}}$. As a difference from the neutral fitness landscape (see Fig. 4), the dynamics becomes extremely simplified. We conjecture that the increase of parasites mutation rates, added to the higher pressure of parasites on hosts because the asymmetry in the fitness landscape would involve parasites to run faster through the sequence space, thus trapping host genotypes 

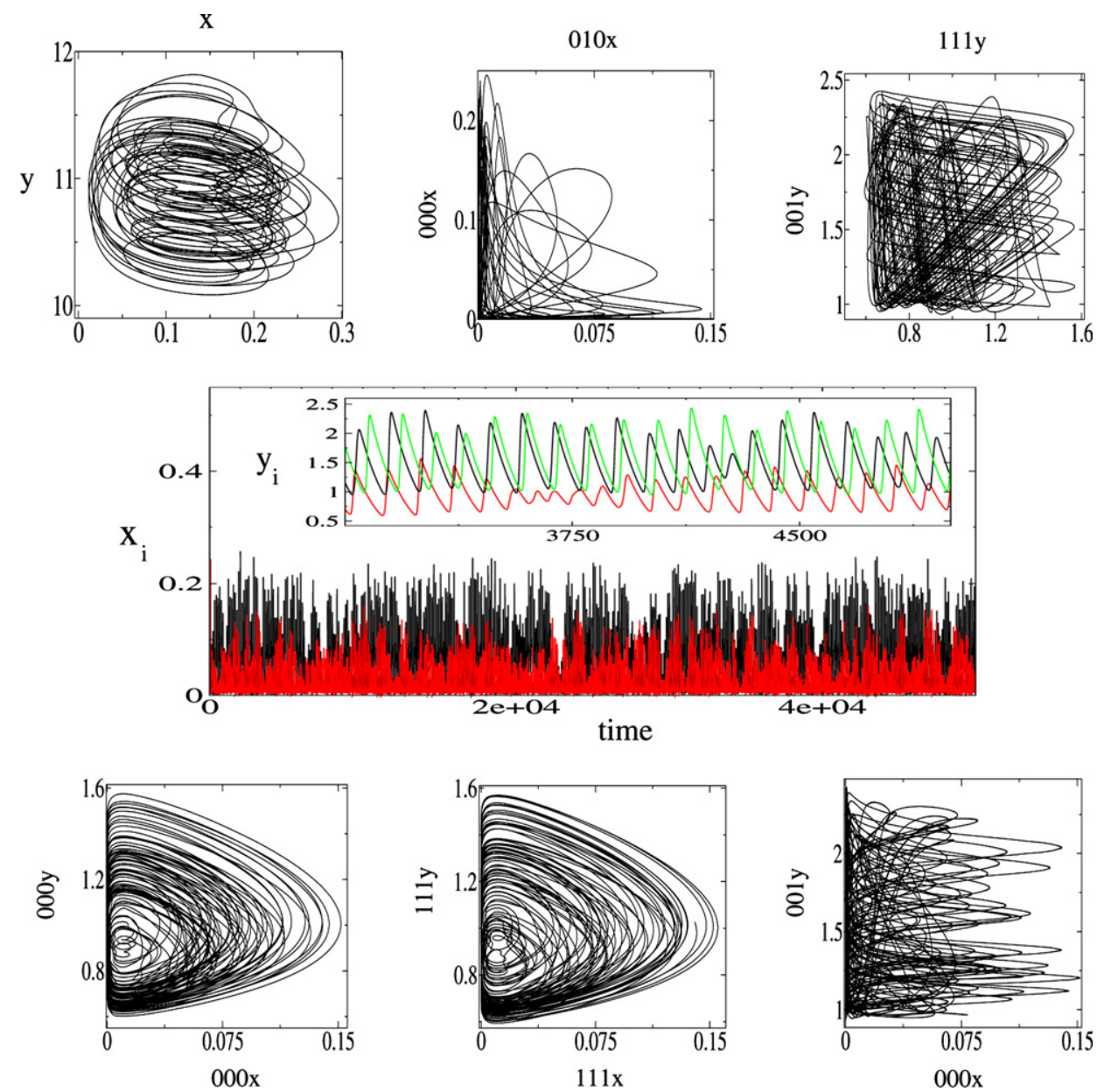

Fig. 6. Chaotic coevolutionary dynamics in the rugged fitness landscape with $k^{\mathrm{p}}=0.6, K^{\mathrm{p}}=0.95$ and $\mu^{\mathrm{h}}=\mu^{\mathrm{p}}=10^{-4}$. The time series plot shows the concentration evolution for both host genotypes 000 (red) and 011 (black). The inset also shows the time evolution for the parasite genotypes 111 (red), 100 (black) and 010 (green). The strange attractors for global and local dynamics are also shown. (For interpretation of the references in colour in this figure legend, the reader is referred to the web version of this article.)

and decreasing their populations. Such an effect, as described in the previous section, would also cause a decrease in the host-parasite time complexity. As seen in Figs. 3-5, the coevolutionary chaotic domain is generically found with low parasites pressure. That is, when both parasite self-replication and/or mutation rates are low. The same effect previously described because of the asymmetry of the fitness landscape is also displayed by the bifurcation diagrams of Fig. 8. Note that here fittest genotypes i.e. 000, are shown to achieve lower maximum values, as opposed to the flattest ones, which show the same bifurcation pattern, now confined in higher concentration values.

\subsection{Parameter space analysis}

The role of parasites pressure in the population dynamics is studied by means of the parameter spaces shown in Fig. 9, where two different mutation scenarios are considered. For the neutral fitness landscape we show that, as seen in the bifurcation diagrams, the increase in parasites self-replication rates involve an initial increase in parasites concentration, however, with $k^{\mathrm{p}} \gtrsim 0.25$ the stationary concentration of the parasite genotypes decreases. Note that for the dynamics of single populations we represent the concentration value for both host-parasite sequences 000, although the same pattern is followed by the other sequences (results not shown). If we now unbalance mutation rates favouring parasite genotypes i.e. here with $\mu^{\mathrm{h}} \ll \mu^{\mathrm{p}}$ (see Fig. 9(c) and (d)), parasite populations are shown to achieve higher sta- 
a
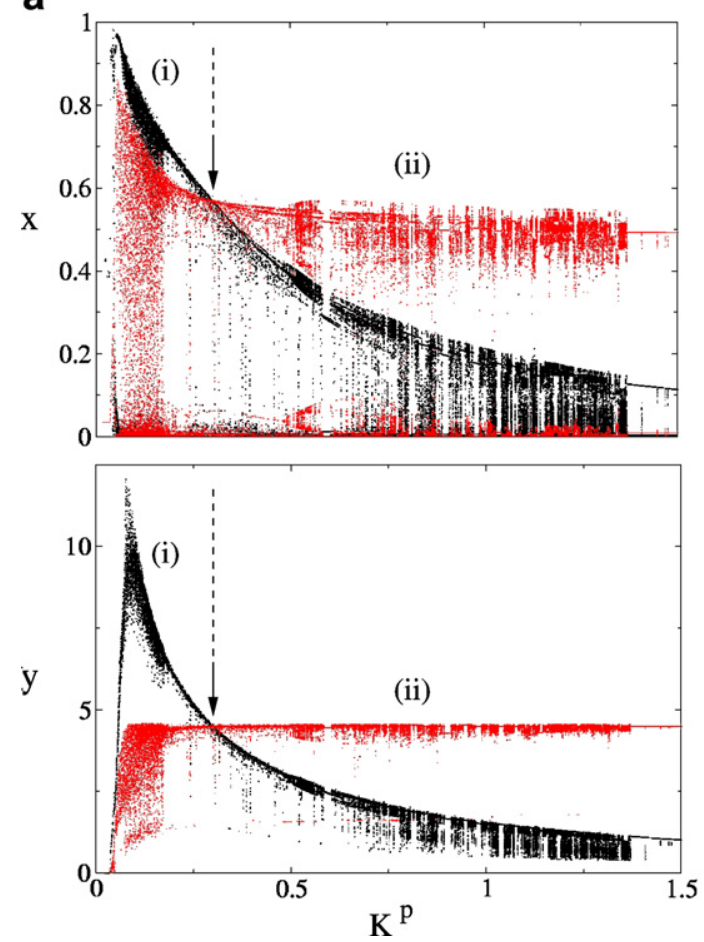

b
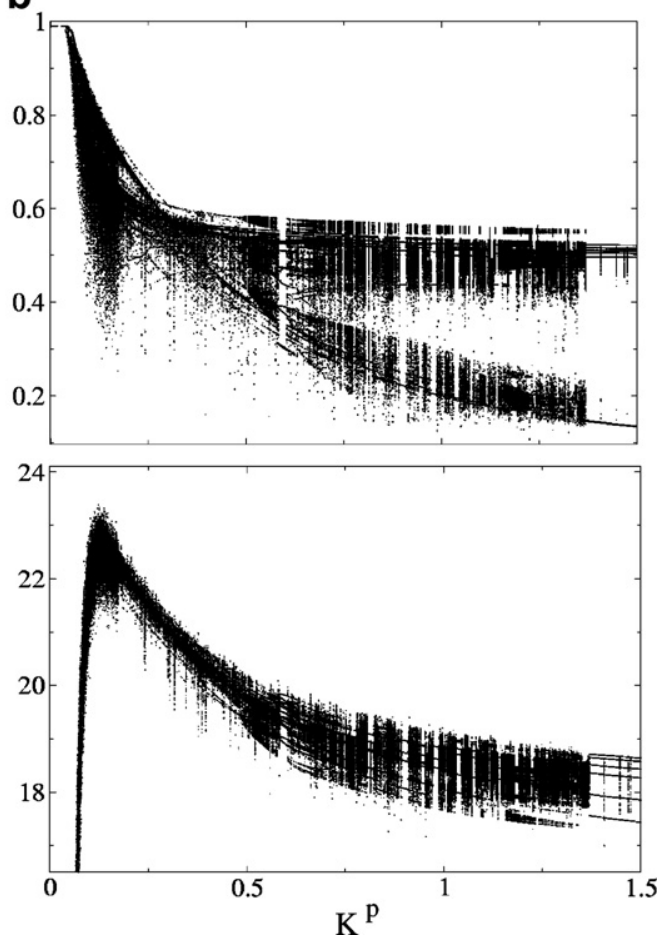

Fig. 7. Bifurcation diagrams for the rugged fitness landscape with $K^{\mathrm{p}}$ as control parameter with $k^{\mathrm{p}}=0.3$ and $\mu^{\mathrm{h}}=\mu^{\mathrm{p}}=10^{-4}$. In $(\mathrm{a})$ we show: host (up) and parasite (down) genotypes 111 (black) and 010 (red). The arrow indicates the point where $K^{\mathrm{p}}=k^{\mathrm{p}}$ i.e. neutral fitness landscape, which separates two different fitness landscapes for parasite genotypes, given by (i) and (ii), where genotypes 111 and 000 are, respectively, flattest and fittest. In (b) the diagrams correspond to the global dynamics for hosts (up) and parasite (down) populations. (For interpretation of the references in colour in this figure legend, the reader is referred to the web version of this article.)

a
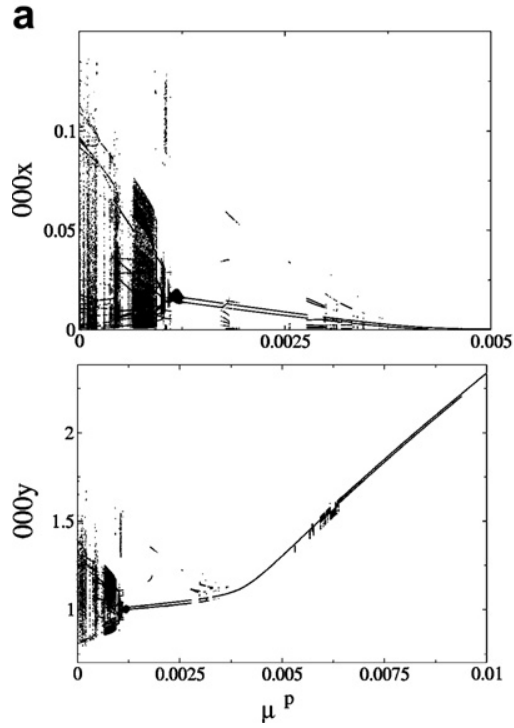

b
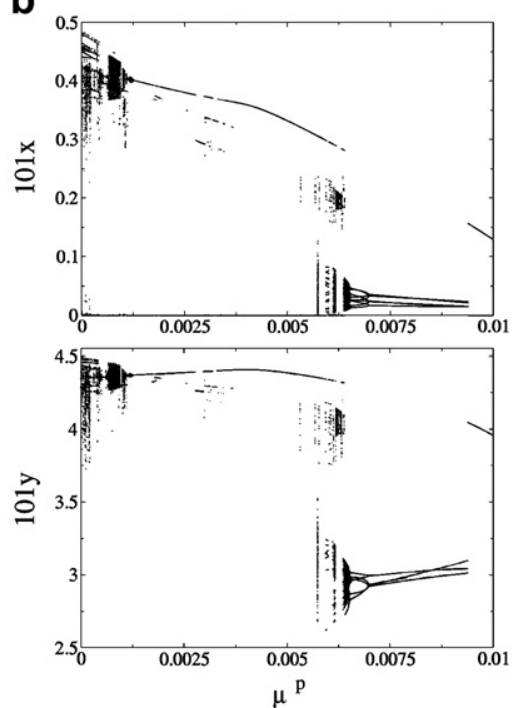
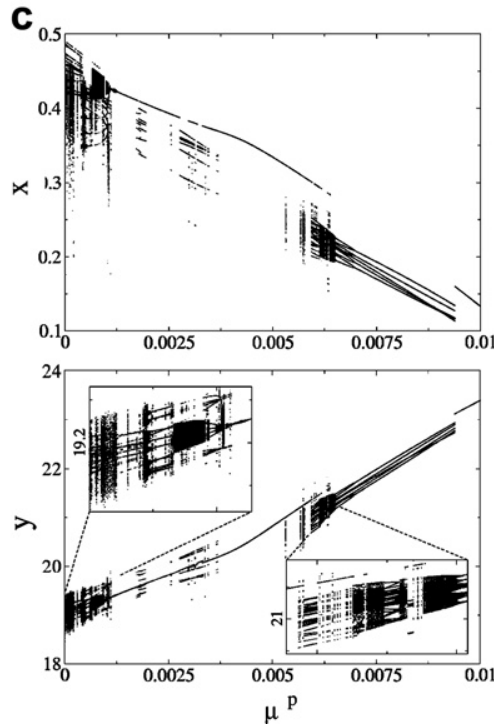

Fig. 8. Bifurcation diagrams for the rugged fitness landscape using $\mu^{\mathrm{p}}$ as control parameter with $k^{\mathrm{p}}=0.3, K^{p}=0.75$ and $\mu^{\mathrm{h}}=10^{-3}$. In (a) we show the dynamics for the host (up) and parasite (down) genotypes 000. In (b) and (c) we show, respectively, the same diagrams for a hypercubic neighboring genotype, and for the global host (up) and parasite (down) populations. 

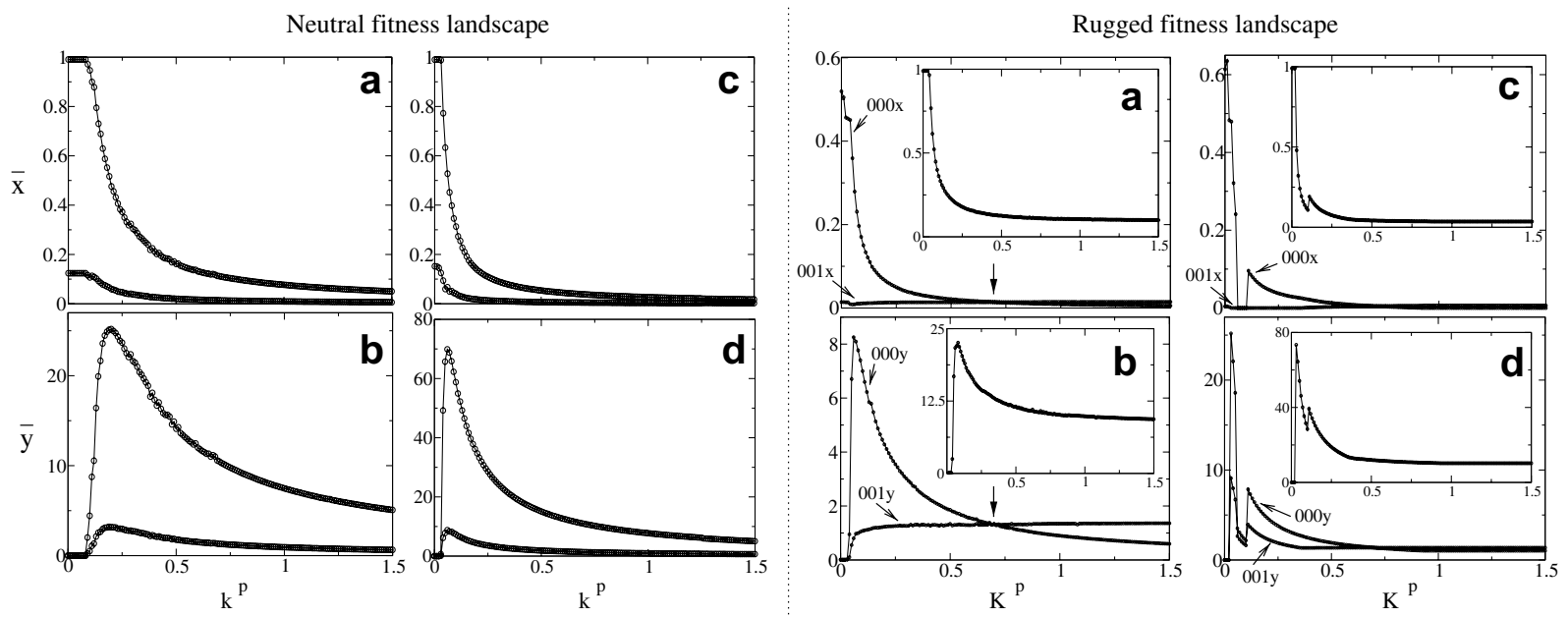

Fig. 9. Parasites self-replication rates in both fitness landscapes. Each data point corresponds to the mean concentration taken over the last $15 \times 10^{4}$ time steps of a time series with $3 \times 10^{5}$ time steps. In both fitness landscapes: (a and b) $\mu^{\mathrm{h}}=\mu^{\mathrm{p}}=10^{-3} ;\left(\mathrm{c}\right.$ and d) $\mu^{\mathrm{h}}=10^{-6}$ and $\mu^{\mathrm{p}}=0.01$. For the neutral case: in (a) and (c) we show the stationary concentration for the host genotype 000 (down) and for the whole population (up). In (b) and (d) we show the same now for the parasite population. For the rugged fitness landscape we repeat the same analyses but now showing the stationary concentration for the sequences 000 and 001 for host (a) and (c) and parasite populations (b) and (d). The vertical arrow indicates the point where $K^{\mathrm{p}}=k^{\mathrm{p}}$ i.e. neutral fitness landscape. The insets show, in the four plots, the stationary concentrations for the whole populations.

tionary concentration values. Furthermore, because of the higher pressure of parasites on hosts, both host-parasite populations start decreasing at a lower value of $k^{\mathrm{p}}$ i.e. from $k^{\mathrm{p}} \gtrsim 0.07$.

The same analysis is also developed for the rugged fitness landscape, and is also shown in Fig. 9. Here we represent the genotype 000 , which self-replicates at rate $K^{\mathrm{p}}$ (here we use this constant as control parameter), and the orthant genotype neighbor 001, which self-replicate at rate $k^{\mathrm{p}}$. By increasing $K^{\mathrm{p}}$ we can analyze scenarios (i) and (ii), where the flattest sequences are shown to achieve higher stationary concentration values. Note that from $K^{\mathrm{p}} \geqslant k^{\mathrm{p}}$, both host-parasite genotypes 000 (which become fittest) decrease their stationary concentration values. A similar pattern is shown to happen when $\mu^{\mathrm{p}} \gg \mu^{\mathrm{h}}$ (Fig. 9(c) and (d)). Note that when $K^{\mathrm{p}}<k^{\mathrm{p}}$, the genotype 000 also achieves a higher concentration value, but the imbalance in mutation rates even shows an extinction case for the genotype 000 . The stationary concentration for the global host-parasite populations in all the analyses is shown to maintain further away from the extinction threshold. However, when parasite mutation rate increases, host global concentration approaches to extinction. On the contrary, the stationary concentration for parasite genotypes becomes higher, although the decrease of the population is sharper (see Fig. 9 for details).

Let us now analyze the role of parasites pressure by means of three-dimensional parameter spaces, plotting parasite self-replication against parasite mutation rates, considering the neutral fitness landscape. The increase of these two parameters might suppose a higher pressure on hosts because parasites rapidly self-replicate, also running faster through the hypercube (because of a higher diffusion i.e. mutation, through the hypercube). In Fig. 10 we show the surface embedded in this parameter space corresponding to the mean concentration (averaged over a long time series on the attractor) for host-parasite genotypes 000 . As previously shown, the increase in parasites pressure (increase in both $k^{\mathrm{p}}$ and $\mu^{\mathrm{p}}$ ) involve a rapid decrease of both host-parasite stationary concentrations. Moreover, in such a figure we also show the host-parasite attractors for the same sequence. A coarse scanning on the concentration surface suggests that the coevolutionary attractors become simpler when parasite pressure increases. The dynamics with higher values for $k^{\mathrm{p}}$ or $\mu^{\mathrm{p}}$ correspond to quasiperiodic attractors or even to point attractors, while for low values of both $k^{\mathrm{p}}$ and $\mu^{\mathrm{p}}$ the dynamics is generically governed by Red Queen strange attractors (see Fig. 10 for details).

We finally analyze the rates of change i.e. mutation rates, for host-parasite genotypes in order to explore the qualitative dynamics of the system also in the simplest fitness landscape. Here, high mutation rates might involve to "run" faster through the hypercube (as the Red Queen and Alice). Hence, lower mutation rates for host sequences might facilitate parasites trapping hosts. On the contrary, high mutation rates for host genotypes might help such genotypes to escape from parasite sequences (this dynamical effects have been described in the dynamics of spatially extended host-parasite sequences [16]). Hence, we now repeat the same study as in Fig. 10, now representing $\mu^{\mathrm{h}}$ against $\mu^{\mathrm{p}}$. 
a

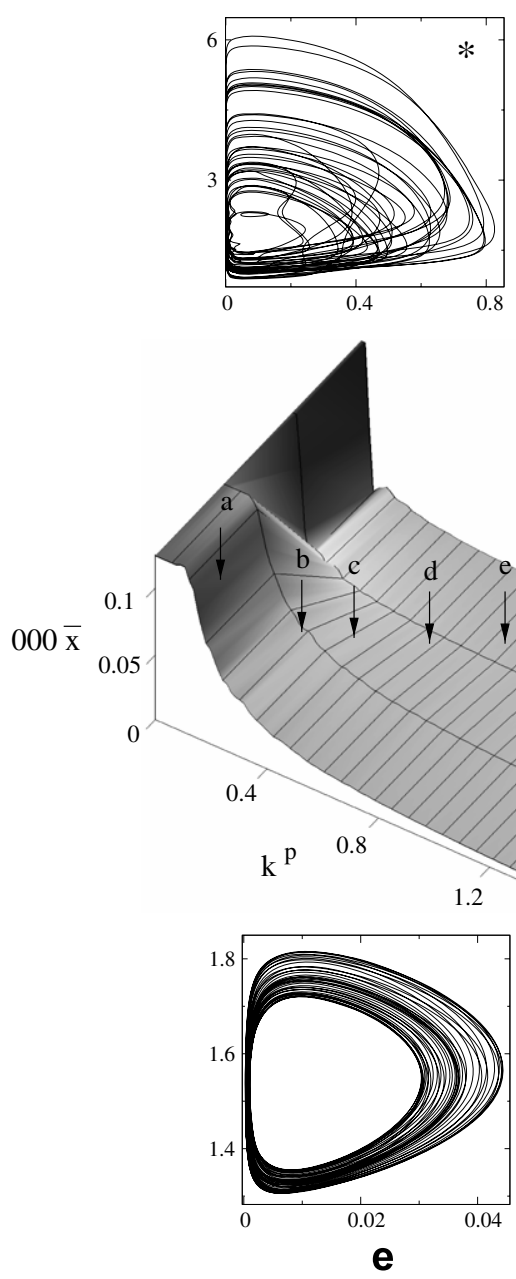

b

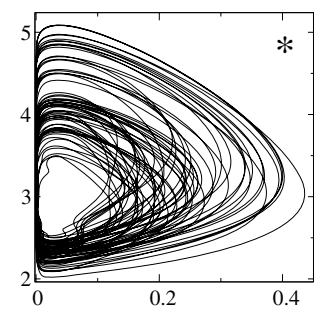

C

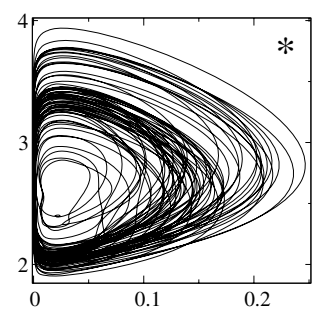

d

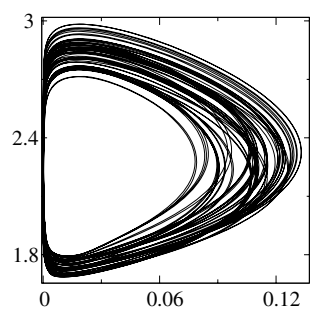

Fig. 10. Parameter space for the neutral fitness landscape by using parasite self-replication, $k^{\mathrm{p}}$, and parasite mutation rate, $\mu^{\mathrm{p}}$, with $\mu^{\mathrm{h}}=10^{-3}$. Here the mean concentration values are computed as in the previous figure. We also show the dynamics for the parameter values indicated with the arrows in projections onto the $(000 x, 000 y)$ phase plane. Note that from (a) to (f) the dynamics becomes simplified, with a qualitative change in the attractors and a transition from turbulent to ordered coevolution. The same trend (i.e. dynamical simplification) is also found along the direction indicated with the arrows drawn on the parameter space at the right, which corresponds to the parasite genotype i.e. $000 y$ (results not shown).

We specifically study this scenario by considering three different values for $k^{\mathrm{p}}$. The increase of this parameter involves the decrease of both host-parasite populations as well as the simplification of the coevolutionary behavior, in agreement with the previous analyses. Moreover, as previously mentioned, the increase in parasites mutation rates involves the simplification of the coevolutionary dynamics, which is shown to change from chaotic to quasiperiodic dynamics, and even to a point attractor. The increase of host mutation rates can also involve this dynamical simplification (see Fig. 11 for details). Our results are in agreement with the work of Schnabl and co-workers [62], who showed that a strange attractor governing the dynamics of a low-dimensional replicator system was very sensitive to mutation processes. These authors actually described a gradual disappearance of complex dynamics with increasing mutation rates.

\section{Discussion}

In the present work we numerically study a continuous, deterministic mean field model describing the dynamics of gene-for-gene coevolution of two panmictic populations of self-replicating bit sequences undergoing mutation and 
a1

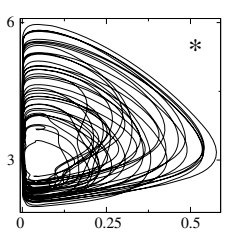

1
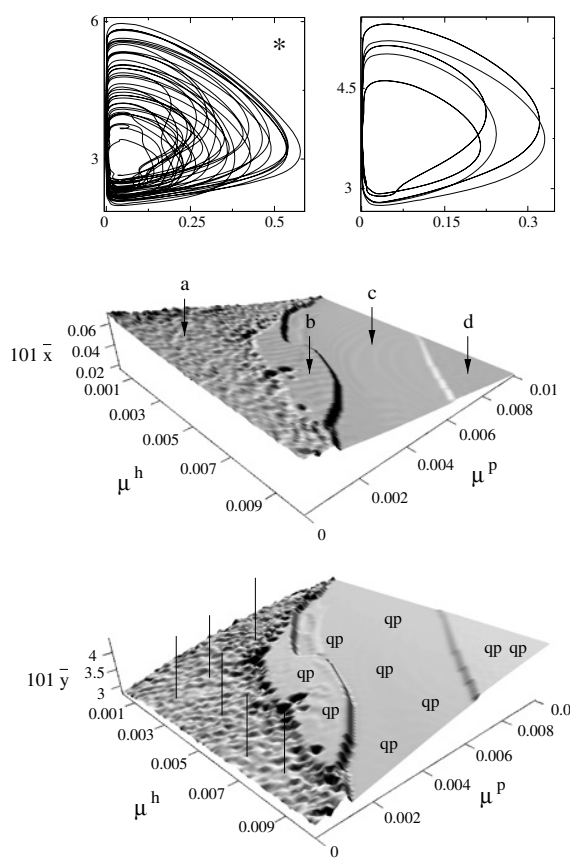

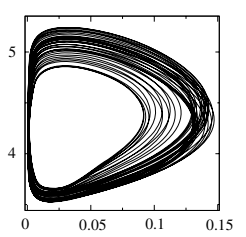

c1

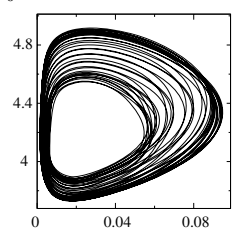

d1 a2

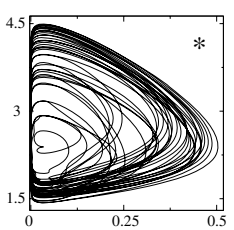

2

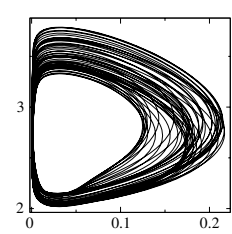

a3

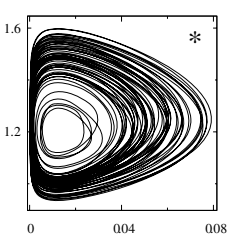

3

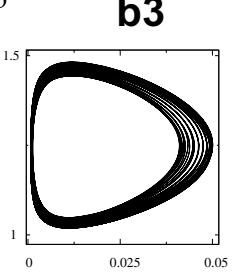

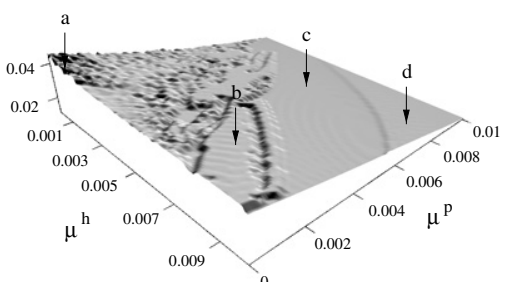
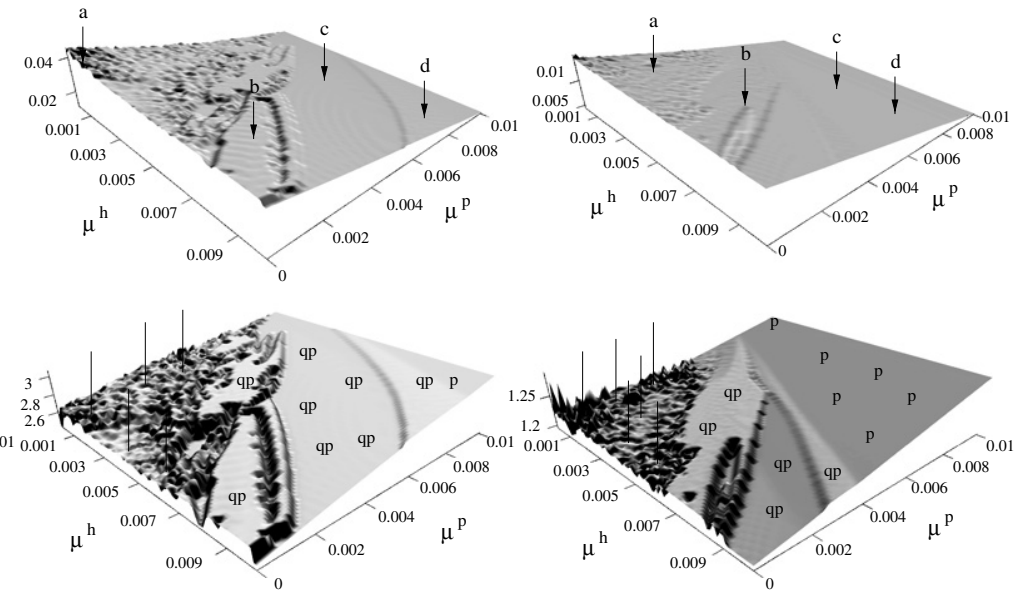

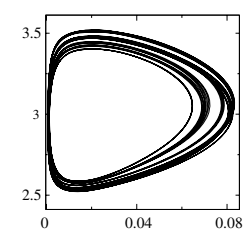

c2

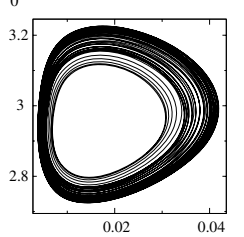

d2

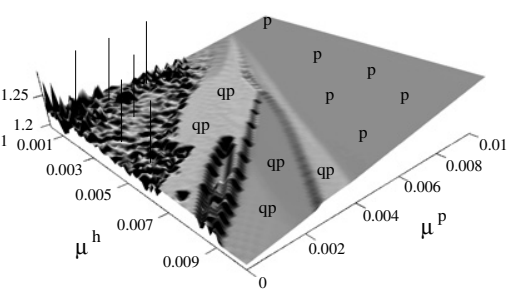

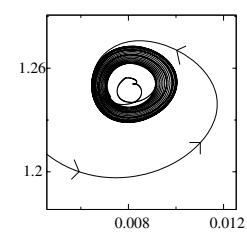

c3

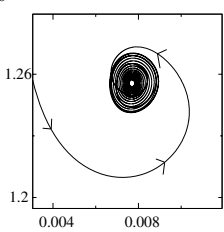

d3

Fig. 11. Same as Fig. 10 now plotting $\mu^{\mathrm{h}}$ against $\mu^{\mathrm{p}}$ for three scenarios with increasing parasite self-replication rates, with (1) $k^{\mathrm{p}}=0.2$ (left), (2) $k^{\mathrm{p}}=0.3$ (middle), and (3) $k^{\mathrm{p}}=0.75$ (right). For each scenario we also represent the attractors projected onto the (101x,101y) phase space, which correspond to the letters on the vertical arrows in the parameter spaces for the host genotype. In the parameter spaces for the parasite sequence $101 y$, we represent, for several combinations of both mutation rates, the dynamics governed by strange (vertical lines), quasiperiodic (qp), and point (p) attractors.

host-parasite interactions. We specifically analyze two different scenarios with parasites evolving in a neutral and in a rugged fitness landscape. For both cases, we show a wide coevolutionary scenario governed by Red Queen strange attractors, in agreement with previous studies about host-parasite interactions $[4,7,11,16]$. In the rugged fitness landscape the fittest genotypes are shown to achieve lower stationary concentrations, as opposed to the flattest ones, which show a higher stationary concentration value. The increase in parasites pressure generically involves a simplification of the dynamical host-parasite behavior, governing the transition from chaotic to quasi-periodic or periodic dynamics. To give advantage to host genotypes (by increasing its mutation rate) can also involve the change from turbulent to ordered coevolution. The possible evolutionary meaning of chaos in population dynamics is a hard question to answer. The ubiquity of chaos in multitude of non-linear ecological models is obvious, and chaos has been even found by using realistic parameters in a model for a three-species food chain [10]. The work of Allen and co-workers [12] elegantly showed that chaos in metapopulations weakly coupled by migration might involve spatial asynchronies able to ensure the persistence of a population under the presence of both internal and external noises, as opposed to the idea of Berryman and Millstein [37], also providing a really suggerent interpretation of ecological chaos from an evolutionary point of view. Other studies have shown that mutation $[7,11]$ as well as the spatial degrees of freedom [16] might act as a stabilizing factors in host-parasite interactions, in the so-called homeochaotic state [7,11].

An interesting question is whether chaos confers some stability advantages to host-parasite populations, as opposed for example to an ordered host-parasite time coupling, which could be more sensible to external perturbations. The 
inherent decorrelation i.e. asynchrony, that chaotic systems often show might probably suppose a robust way to avoid extinctions in population dynamics. Our model suggests that the increase in parasites pressure might have two effects on the host-parasite dynamics. The first one might be a decrease in both host-parasite concentrations (or population densities), thus being the system more sensible to extinction. The other effect might be a qualitative change in the host-parasite dynamics, in which such a simplification could also involve a higher extinction probability. In this sense, the ergodic fashion of chaotic trajectories in phase space could provide interacting species with relevant selective properties, as a great diversity of populational densities or concentration states, able to ensure a sustained dynamical stability by means of diffusive processes as mutation or spatial diffusion, which might serve as a recovering factor under the presence of perturbations. These ideas will be explored in future research.

\section{Acknowledgments}

The authors want to thank Charles L. Dodgson for useful discussions and suggestions. This work has been supported by an EU PACE grant to $J S$ within the 6th Framework Program under contract FP6-002035 (Programmable Artificial Cell Evolution) and by the Santa Fe Institute.

\section{References}

[1] Schaffer WM, Kot M. Chaos in ecological systems: the coals that newcastle forgot. Tree 1986;1(3):58-63.

[2] May R. Simple mathematical models with very complicated dynamics. Nature 1976;261:459-67.

[3] Gilpin ME. Spiral chaos in a predator-prey model. Am Natural 1979;107:306-8.

[4] Seger J. Dynamics of simple host-parasite models with more than two genotypes in each species. Philos Trans Roy Soc Lond B 1988;319:541-55.

[5] Kwiatkowski D, Nowak M. Periodic and chaotic host-parasite interactions in human malaria. Proc Natl Acad Sci USA 1991;88:5111-3.

[6] Hassell MP, Comins NH, May RM. Spatial structure and chaos in insect population-dynamics. Nature 1991;353:255-8.

[7] Kaneko K, Ikegami T. Homeochaos: dynamic stability of a symbiotic network with population dynamics and evolving mutation rates. Physica D 1992;56:406-29.

[8] Solé RV, Bascompte J, Valls J. Nonequilibrium dynamics in lattice ecosystems: chaotic stability and dissipative structures. Chaos 1992;2:387-95.

[9] Solé RV, Valls J, waves Spiral. chaos and multiple attractors in lattice models of interacting populations. Phys Lett A 1992;166:123-8.

[10] Hastings A, Powell T. Chaos in a three-species food chain. Ecology 1991;72(3):896-903.

[11] Ikegami T, Kaneko K. Evolution of host-parasitoid network through homeochaotic dynamics. Chaos 1992;2(3):397-407.

[12] Allen JC, Schaffer WM, Rosko D. Chaos reduces species extinction by amplifying local population noise. Nature 1993;364:229-32.

[13] Dhamala M, Lai Ying-Cheng. Controlling transient chaos in deterministic flows with applications to electrical power systems and ecology. Phys Rev E 1999;59(2):646-1655.

[14] Huisman J, Weissing FJ. Biodiversity of plankton by species oscillations and chaos. Nature 1999;402:407-10.

[15] Gamarra JGP, Solé RV. Bifurcations and chaos in ecology: lynx returns revisited. Ecol Lett 2000;3:0-8.

[16] Sardanyés J, Solé RV. Chaotic stability in spatially-resolved host-parasite replicators: the Red Queen on a lattice. Int J Bifurcat Chaos, in press.

[17] Forst CV. Chaotic interactions of self-replicating RNA. Comput Chem 1996;20(1):69-83.

[18] Upadhyay Ranjit Kumar, Rai Vikas. Why chaos is rarely observed in natural populations. Chaos, Solitons \& Fractals 1997;8(12):1933-9.

[19] Rai V, Schaffer WM. Chaos in ecology. Chaos, Solitons \& Fractals 2001;12:197-203.

[20] Schaffer WM. Order and chaos in ecological system. Ecology 1985;66:93-106.

[21] Strogatz SH. Nonlinear dynamics and chaos with applications to physics, biology, chemistry, and engineering. Westview Press; 2000.

[22] Lorenz EN. Deterministic nonperiodic flow. J Atmos Sci 1963;20:130-41.

[23] Rössler OE. An equation for continuous chaos. Phys Lett 1976;57:397-8.

[24] Gollub JP, Swinney HL. Onset of turbulence in a rotating fluid. Phys Rev Lett 1975;35:927-30.

[25] Bergé P, Dubois M, Manneville P, Pomeau Y. Intermittency in Rayleigh-Bénard convection. Part II: Experiments, in universality in chaos. Bristol and New York: Adam Hilger; 1980.

[26] Libchaber A, Maurer J. A Rayleigh-Bénard experiment: helium in a small box. Part II: Experiments, in universality in chaos. Bristol and New York: Adam Hilger; 1989. 
[27] Libchaber A, Laroche C, Fauve S. Period doubling cascade in mercury, a quantitative measurement. Part II: Experiments, in Universality in chaos. Bristol and New York: Adam Hilger; 1982.

[28] Arecchi FT, Meucci R, Puccioni G, Tredicce J. Experimental evidence of subharmonic bifurcations, multistability, and turbulence in a $Q$-switched gas laser. Phys Rev Lett 1982;49:1217-20.

[29] Testa J, Pérez J, Jeffries C. Evidence for universal chaotic behavior of a driven nonlinear oscillator. Phys Rev Lett 1982;48:714-7.

[30] Hastings A, Hom Carole L, Ellner S, Turchin P, Charles H, Godfray J. Chaos in ecology: is mother nature a strange attractor? Annu Rev Ecol Syst 1993;24:1-33.

[31] Argoul F, Arneodo A, Richetti P, Roux JC. Chemical chaos: from hints to confirmation. Acc Chem Res 1987;20:436-42.

[32] Scott Stephen K. Chemical chaos. International series of monographs on chemistry, vol. 24. Oxford: Clarendon Press; 1994.

[33] Roux JC, Rossi A, Bachelart S, Vidal C. Representation of a strange attractor from an experimental study of chemical turbulence. Physica D 1983;8:257-66.

[34] Hudson JL, Mankin JC. Chaos in the Belousov-Zhabotinski reaction. J Chem Phys 1981;74:6171-7.

[35] Roux JC, Simoyi RH, Swinney HL. Observation of a strange attractor. Physica D 1983;8:257-66.

[36] Hassell MP, Lawton JH, May RM. Patterns of dynamical behavior in single species population models. J Anim Ecol 1976;45:471-86.

[37] Berryman AA, Millstein JA. Are ecological systems chaotic - and if not, why not? Tree 1986;4:26.

[38] Turchin P, Taylor AD. Complex dynamics in ecological time series. Ecology 1992;73(1):289-305.

[39] Solé RV, Bascompte J. Self-organization in complex ecosystems. Monographs in population biology. Princeton: Princeton University Press; 2006.

[40] Cushing JM, Constantino RF, Dennis Brian, Desharnais RA, Henson Shandelle M. Nonlinear population dynamics: models, experiments and data. J Theor Biol 1998;194:1-9.

[41] Poole Robert W. Periodic, pseudoperiodic, and chaotic population fluctuations. Ecology 1977;58:210-3.

[42] Tilman D, Wedin D. Oscillations and chaos in the dynamics of a perennial grass. Nature 1991;353:653-5.

[43] Sugihara G, May Robert M. Nonlinear forecasting as a way of distinguishing chaos from measurement error in time series. Nature 1990;344:734 41.

[44] Dieckmann U, Marrow P, Law R. Evolutionary cycling in predator-prey interactions: population dynamics and the Red Queen. J Theor Biol 1995;176:91-102.

[45] Sasaki A, Godfray HCJ. A model for the coevolution of resistance and virulence in coupled host-parasitoid interactions. Proc Roy Soc Lond 1999;266:455-63.

[46] Kamp C, Wilke CO, Adami C, Bornholdt S. Viral evolution under the pressure of an adaptive immune system: optimal mutation rates for viral escape. Complexity 2003;8(2):28-33.

[47] Thompson John N, Burdon Jeremy J. Gene-for-gene coevolution between plants and parasites. Nature 1992;360:121-5.

[48] Gupta S, Ferguson N, Anderson R. Chaos, persistence, and evolution of strain structure in antigenically infectious agents. Science 1998;280:912-5.

[49] Case TJ. An illustrated guide to theoretical ecology. Predator-prey systems: predator dynamics and effects on prey. Oxford (New York): Oxford University Press; 2000.

[50] Solé RV, Bascompte J. Self-organization in complex ecosystems. Nonlinear dynamics. Monographs in population biology. Princeton University Press; 2006.

[51] Van Valen L. A new evolutionary law. Evol Theory 1973;1:1.

[52] Van Valen L. Two modes of evolution. Nature 1974;252:298-300.

[53] Hoffman A. Testing the Red Queen hypothesis. J Evol Biol 1991;4:1-7.

[54] Nowak MA, Sigmund K. Evolutionary dynamics of biological games. Science 2004;303(6):793-9.

[55] Lewis Carroll, Through the looking-glass and what Alice found there (Reprinted in Alice in Wonderland, W.W. Norton Co. Inc., NY, 1971). 1871.

[56] Stenseth Nils Chr. Where have all the species gone? On the nature of extinction and the Red Queen hypothesis. OIKOS 1979;33:196-227.

[57] Bell G. The masterpiece of nature: the evolution and genetics of sexuality. Berkeley, CA: University of California Press; 1982.

[58] Lively CM. Evidence from a New Zealand snail for the maintenance of sex by parasitism. Nature 1987;328:519-21.

[59] Bell G, Smith J Maynard. Short-term selection for recombination among mutually antagonistic species. Nature 1987;328:66-8.

[60] Hamilton WD, Axelrod R, Tanese R. Sexual reproduction as an adptation to resist parasites (a review). PNAS 1990;87:3566-73.

[61] Stadler BMR, Stadler PF. Molecular replicator dynamics. Adv Complex Syst 2003;6:47-77.

[62] Schnabl W, Stadler PF, Forst C, Schuster P. Full characterization of a strange attractor. Chaotic dynamics in low-dimensional replicator systems. Physica D 1991;48:65-90. 